\title{
An anomalous diffraction study of $\mathrm{Cu}_{2} \mathrm{Zn}(\mathrm{Ge}, \mathrm{Si}) \mathrm{Se}_{4}$
}

\author{
D. M. Többens, G. Gurieva, S. Niedenzu, G. Schuck, S. Schorr \\ Helmholtz-Zentrum Berlin für Materialien und Energie (HZB), Department Structure and Dynamics of Energy Materials, Hahn- \\ Meitner-Platz 1, 14109 Berlin, Germany
}

While the silicon-rich members of the series $\mathrm{Cu}_{2} \mathrm{Zn}(\mathrm{Ge}, \mathrm{Si}) \mathrm{Se}_{4}$ crystallize in wurtz-kesterite type structure [1], germanium-rich samples adopt a tetrahedral structure of the kesterite type [2] (figure 1). Identification of the silicon site is straightforward from regular X-ray diffraction, as $\mathrm{Si}^{4+}$ is a light element and has less electrons than the other cations. However, $\mathrm{Cu}^{1+}, \mathrm{Zn}^{2+}$, and $\mathrm{Ge}^{4+}$ are all isoelectronic and have very similar form factors. The kesterite type of the cation distribution of $\mathrm{Cu}_{2} \mathrm{ZnGeSe}_{4}$ has been established by neutron diffraction [2], which can distinguish these elements.

We now applied anomalous X-ray diffraction to this system, using Rietveld refinement and Multiple Edge Anomalous Diffraction (MEAD) [3] with data taken at the K-absorption edges of $\mathrm{Cu}, \mathrm{Zn}$, and $\mathrm{Ge}$. These energies are accessible at beamline KMC-2, BESSY II, Berlin [4]. The Si-rich end member $\mathrm{Cu}_{2} \mathrm{ZnSiSe}_{4}$ has previously shown to be wurtz-kesterite by MEAD [1]. With the correct structure type, the degree of $\mathrm{Cu} / \mathrm{Zn}$ disorder (see caption figure 1) within the Si-rich region of the series could be determined reliably from multiple-energy Rietveld refinement. For the Ge-rich, tetragonal structures, MEAD was found to be the method of choice. In contrast to previous studies, where $\mathrm{Sn}^{4+}$ was the $\mathrm{M}(\mathrm{IV})$ species in the structure [1], in $\mathrm{Cu}_{2} \mathrm{ZnGeSe}_{4}$ all cations have very similar scattering power under normal conditions. This results in superstructure peaks (with respect to the cubic $\mathrm{ZnS}$ parent structure) that are very weak. For Rietveld analysis this is a drawback, as the optimization will be dominated by the main peaks of the parent structure. In MEAD, however, it increases the effect of the changing scattering power close the absorption edges. As a result, not only are Kesterite and Stannite types clearly distinguishable at the $\mathrm{Cu}-\mathrm{K}$ edge (figure 2), also the $\mathrm{Cu} / \mathrm{Zn}$ ordering within the Kesterite structure is clearly detectable and quantifiable at the $\mathrm{Zn}-\mathrm{K}$ edge.

The degree of $\mathrm{Cu} / \mathrm{Zn}$ order for the full series could thus be compared to other structural and physical parameters within the $\mathrm{Cu}_{2} \mathrm{Zn}(\mathrm{Ge}, \mathrm{Si}) \mathrm{Se}_{4}$ solid solution series.

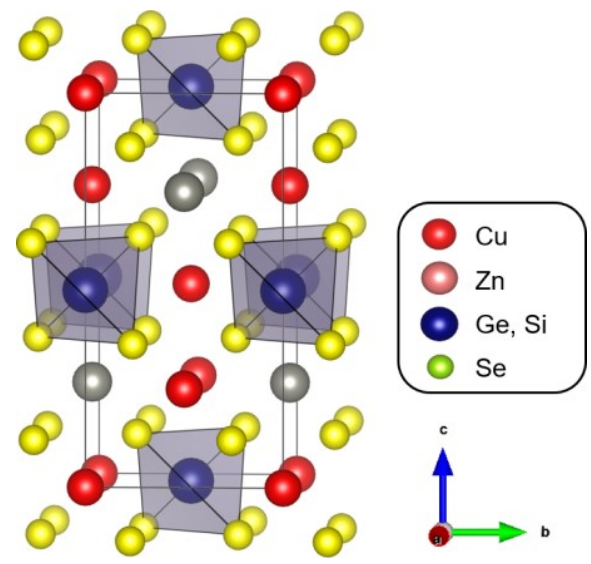

Figure 1. Kesterite structure with fully ordered $\mathrm{Cu} / \mathrm{Zn}$ layers. Disorder affects cation distribution in $\mathrm{Cu} / \mathrm{Zn}$ layers. Stannite type would have pure $\mathrm{Cu}$ layers and $\mathrm{Zn} /(\mathrm{Ge}, \mathrm{Si})$ layers. Wurtzkesterite type structure is comparable in this regard.

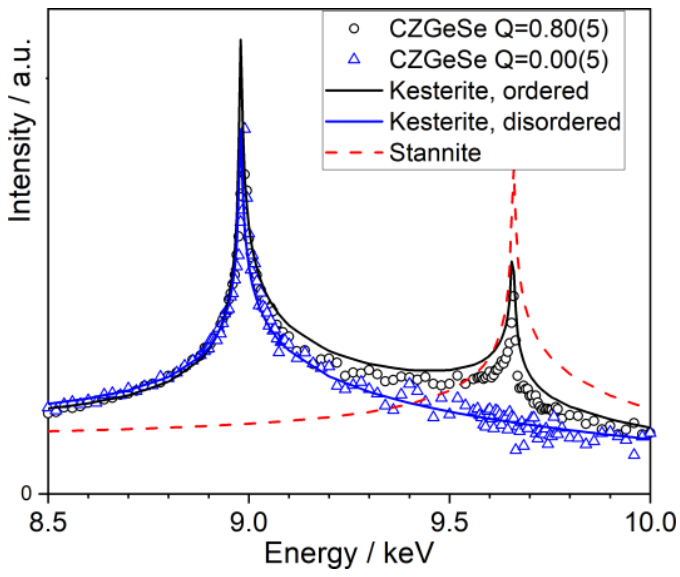

Figure 2. Observed and simulated MEAD spectra of Bragg peak 011. Samples are Kesterite type with high degree of $\mathrm{Cu} / \mathrm{Zn}$ order $\left(\mathrm{Cu}_{2.06} \mathrm{Zn}_{0.99} \mathrm{Ge}_{0.99} \mathrm{Se}_{4}\right)$ and disordered $\left(\mathrm{Cu}_{2.28} \mathrm{Zn}_{0.95} \mathrm{Ge}_{0.96} \mathrm{Se}_{4}\right)$, respectively.

[1] Többens, D. M., Gurieva, G., Niedenzu, S., Schuck, G., Schorr, S. (2020) Acta Cryst. B 76, 1027.

[2] Gurieva, G., Többens, D. M., Valakh, M. Y., Schorr, S. (2016) J. Phys. Chem. Solids 99, 100.

[3] Collins, B. A., Chu, Y. S., He, L., Haskel, D. \& Tsui, F. (2015). Phys. Rev. B 92, 224108.

[4] Helmholtz-Zentrum Berlin für Materialien und Energie (2016) J. Large-Scale Res. Facilities 2, A49

Keywords: Kesterite; semiconductor; anomalous scattering; MEAD; cation disorder

This work was made possible by allocation of synchrotron radiation beamtime at BESSY II, Helmholtz-Zentrum Berlin (HZB).

Acta Cryst. (2021), A77, C1064 